\title{
Inversão da forma de onda completa em sismogramas de reflexão rasa
}

Allan Segovia Spadini'; Liliana Alcazar Diogo'1, Renato Luiz Prado'1.

${ }^{1}$ Instituto de Astronomia, Geofísica e Ciências Atmosféricas - Universidade de São Paulo.

Copyright 2014, SBGf - Sociedade Brasileira de Geofísica

Este texto foi preparado para a apresentação no VI Simpósio Brasileiro de Geofísica, Porto Alegre, 14 a 16 de outubro de 2014. Seu conteúdo foi revisado pelo Comitê Técnico do VI SimBGf, mas não necessariamente representa a opinião da SBGf ou de seus associados. É proibida a reprodução total ou parcial deste material para propósitos comerciais sem previa autorização da SBG.

\section{Resumo}

Neste trabalho um algoritmo de inversão da forma de onda completa é avaliado sobre dados de sísmica rasa, até 100 metros de profundidade. O algoritmo proposto busca uma solução global do modelo 1D de velocidades de ondas S e densidades. Foram obtidos bons resultados sobre modelos sintéticos para espaços de busca bem definidos. Para a aplicação sobre dados reais ainda precisa ser melhor avaliada a wavelet utilizada.

\section{Introdução}

O processamento de dados de sísmica de reflexão rasa através de técnicas convencionais é um problema desafiador. Grande parte dos eventos de reflexão é mascarado pela presença de ruído coerente como o ground roll. Além disso, reflexões relacionadas ao topo rochoso podem estar muito próximas aos eventos de refração, dificultando a sua interpretação.

Nesta escala de investigação dados de refração e dados adquiridos para a técnica de análise multicanal de ondas superficiais (MASW) são largamente utilizados. E diversos autores como Ditmar et al. (1999), Vesnaver et al. (1999), Grandjean (2006) e Fabien-ouellet e Fortier (2014) tem empregado mais de um tipo de evento na análise e inversão de sismogramas nesta escala. Neste sentido, o ruído coerente eliminado no processamento de reflexão pode ser utilizado como informação adicional.

Inversão da forma de onda completa (FWI) é um problema de ajuste de dados sintéticos aos dados observados, considerando o campo de onda completo. Na escala de sísmica rasa podem ser citados os trabalhos de Gélis, Virieux e Grandjean (2007), Romdhane et al. (2008) e Romdhane et al. (2009), tendo sido aplicados com eficiência apenas a dados sintéticos e utilizando técnicas diferentes. Além disso, FWI representa um problema mal posto com múltiplos pontos de mínimo, que requer uma boa aproximação inicial. Vale ressaltar também que a inversão de dados $2 \mathrm{D}$ e $3 \mathrm{D}$ requer muito tempo computacional.

Uma forma alternativa trabalhada em Liu e Zhang (2013) foi a utilização de um algoritmo de busca 1D em conjuntos CMP, para a obtenção de soluções mais rápidas. Outro aspecto é a possibilidade em uma busca 1D é utilização de algoritmos globais de busca. Em especial, algoritmos genéticos não precisam de uma boa estimativa inicial e requerem apenas que o tamanho do espaço de busca dos parâmetros do modelo seja definido.
A aproximação 1D é a mesma realizada no processamento de refração/reflexão convencional. Desta forma, é interessante analisar a possiblidade de extração de parâmetros como a velocidade da onda $S$ e as densidades do meio, já que estes parâmetros também são utilizados na construção dos sismogramas através das equações elastodinâmicas.

Neste trabalho é considerado um problema elástico de inversão da forma de onda completa. Desta forma, será realizada uma busca global através da utilização de algoritmos genéticos. Para a análise da técnica a metodologia foi aplicada a dados sintéticos simulando um conjunto de tiro com um grande intervalo de afastamentos. E também foram utilizados dados reais com situação próxima a simulada no caso sintético obtidos no campus da Universidade de São Paulo.

\section{Metodologia}

Foi considerada uma formulação elástica para o problema direto. A modelagem dos dados sísmicos foi realizada com o código produzido em Thorbecke e Draganov (2011) que utiliza um algoritmo de diferenças finitas de malha deslocada como o proposto por Virieux (1986), porém com precisão temporal de segunda ordem e espacial de quarta ordem.

A função objetivo utilizada considera a norma quadrática da diferença entre o dado observado e o sintético:

$$
f_{\text {objetivo }}=\left[\sum_{i} \sum_{j}\left(s_{i j}^{\text {observado }}-s_{i j}^{\text {calculado }}\right)^{2}\right],
$$

onde s representa o traço sísmico, i é o índice que representa a amostra em cada traço, j o índice que percorre os traços do conjunto.

O algoritmo genético trabalha tentando minimizar a equação (1). O método é aplicado considerando um modelo com um número de camadas pré-definido onde as velocidades da onda $\mathrm{P}$ e as profundidades dos refletores são conhecidas e as velocidades da onda $S$ e as densidades são as incógnitas.

\section{Resultados com Dados Sintéticos}

Os testes sobre dados sintéticos foram realizados sobre um conjunto de tiro construído utilizando o modelo da Tabela 1 que pode ser visualizado na Figura 1. Para o conjunto de tiro sintético foi adotado um afastamento mínimo de um metro e máximo de 96 metros. O tempo de registro foi de um segundo e o intervalo de amostragem foi de 0,0005 segundos. A wavelet adotada é uma wavelet de Gauss de fase zero com frequência central de $50 \mathrm{~Hz}$. No procedimento de inversão foi utilizada a mesma wavelet do dado da Figura 1. 
Tabela 1: Modelo utilizado na construção do conjunto de tiro sintético.

\begin{tabular}{|c|c|c|c|c|}
\hline Camada & Profundidade $(\mathrm{m})$ & $V_{p}(\mathrm{~m} / \mathrm{s})$ & \multicolumn{2}{|c|}{} \\
\hline 1 & 5 & 370 & 112 & 1530 \\
\hline 2 & 35 & 1650 & 312 & 1910 \\
\hline 3 & - & 4200 & 1680 & 2540 \\
\hline
\end{tabular}

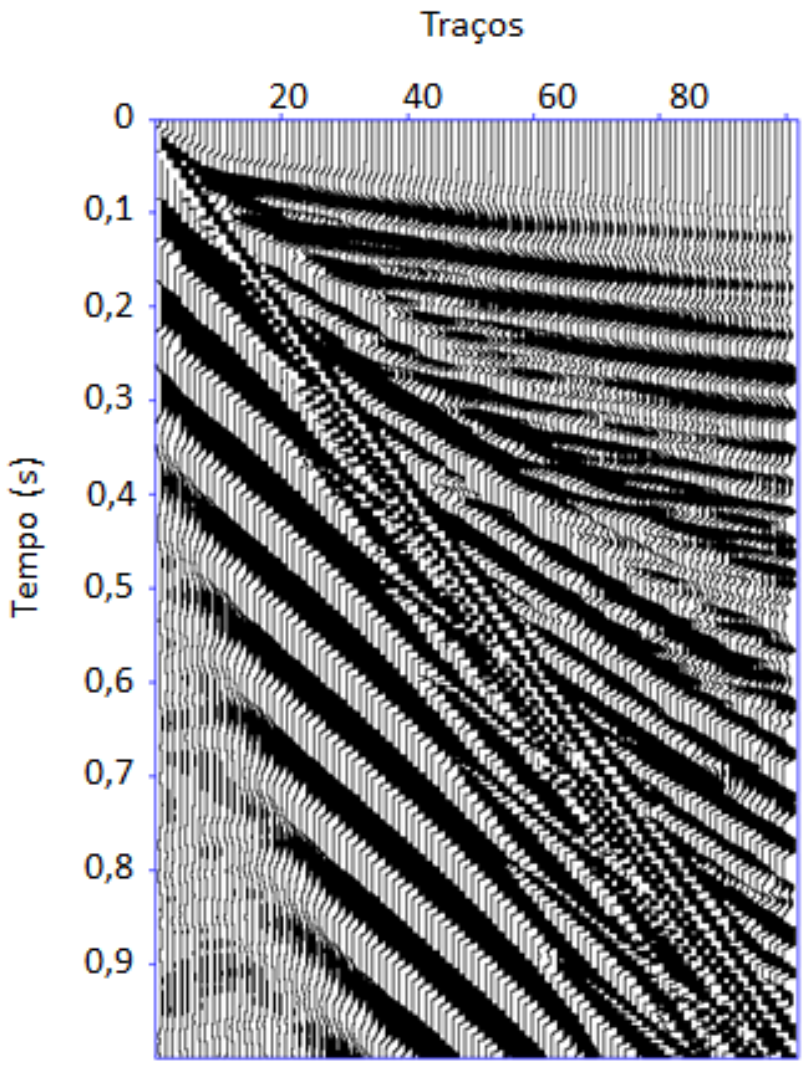

Figura 1: Conjunto de tiro gerado para os testes sintéticos.

Para o algoritmo genético foram definidos os intervalos de busca de velocidades e densidades possíveis (Tabela 2 ). Os limites inferiores e superiores de busca foram definidos para cada camada individualmente. Como um vínculo, o espaço de busca de cada camada foi definido de forma a favorecer resultados onde os valores aumentassem com a profundidade. Para que houvesse convergência, na primeira camada foi necessário restringir bastante o intervalo de velocidades da onda $\mathrm{S}$. Isto porque a presença do evento linear no sismograma da Figura 1, que parte do zero e chega no instante de 1s no traço 96, está relacionado a baixas velocidades da onda $S$.

Para melhorar a convergência a população inicial do algoritmo genético foi alimentada com 40 soluções iniciais aleatórias que respeitassem os vínculos definidos pelos intervalos de busca e de valores dos parâmetros crescendo com a profundidade.
Tabela 2: Intervalos utilizados na busca do algoritmo genético.

\begin{tabular}{|c|c|c|}
\hline Camada & Vs $(\mathrm{m} / \mathrm{s})$ & $\rho\left(\mathrm{Kg} / \mathrm{m}^{3}\right)$ \\
\hline 1 & $110-200$ & $1000-2000$ \\
\hline 2 & $200-800$ & $1500-3000$ \\
\hline 3 & $800-2000$ & $2000-3000$ \\
\hline
\end{tabular}

Foram utilizados 40 elementos para o tamanho da população em cada geração e um valor máximo de 110 gerações. O algoritmo avalia as 110 gerações ou para antes caso a função objetivo pare de mudar. Os valores estimados para as velocidades da onda $S$ e para as densidades podem ser observados na Tabela 3. A estimativa foi realizada diversas vezes sobre o mesmo conjunto de dados com ruído adicionado. Foi utilizada uma razão sinal ruído de 60 .

Tabela 3: Valores médios dos resultados obtidos para a estimativa sobre o dado real e respectivos desvios padrão.

\begin{tabular}{|c|c|c|c|c|}
\hline Camada & Vs (m/s) & $\begin{array}{c}\text { Desvio } \\
\text { Padrão de Vs }\end{array}$ & $\rho\left(\mathrm{Kg} / \mathrm{m}^{3}\right)$ & $\begin{array}{c}\text { Desvio } \\
\text { Padrão de } \rho\end{array}$ \\
\hline 1 & 141,2 & 40,0 & 1905,4 & 120,4 \\
\hline 2 & 274,7 & 23,2 & 2304,8 & 563,2 \\
\hline 3 & 1667,1 & 316,4 & 2585,2 & 238,1 \\
\hline
\end{tabular}

\section{Resultados Sobre Dados Reais}

Para os testes com dados reais foi utilizado um conjunto de tiro semelhante ao apresentado nos testes sintéticos. $O$ conjunto de tiro apresentado (Figura 2) foi adquirido no trabalho de Granda (2010) para análise de ruído. A aquisição foi realizada no terreno do Instituto de Física da Universidade de São Paulo. A geologia local é representada por sedimentos terciários da Formação Itaquaquecetuba da Bacia Sedimentar de São Paulo. Em levantamentos previamente realizados na região como em Porsani et al. (2004) e Diogo, Le Diagon e Prado (2004) são observadas interfaces aproximadamente planas e um topo rochoso em aproximadamente 53 metros de profundidade. A fonte utilizada foi uma marreta de $7 \mathrm{Kg}$ com afastamento de um metro do primeiro geofone. O arranjo possui 96 geofones espaçados de um metro cada.

Para o caso real a wavelet também é uma incógnita. Considerando as características da fonte e do espectro do dado real foi construída uma wavelet de ricker de frequência central de $100 \mathrm{~Hz}$. Esta wavelet foi transformada em uma wavelet de fase mínima a partir da técnica de Kolmogorov. Para a velocidade da onda $\mathrm{P}$ e profundidades foram considerados os trabalhos de Granda (2010), Porsani et al. (2004) e Diogo et al. (2004) onde as velocidades e profundidades foram estimadas a partir das primeiras chegadas e do processamento do evento de reflexão. 


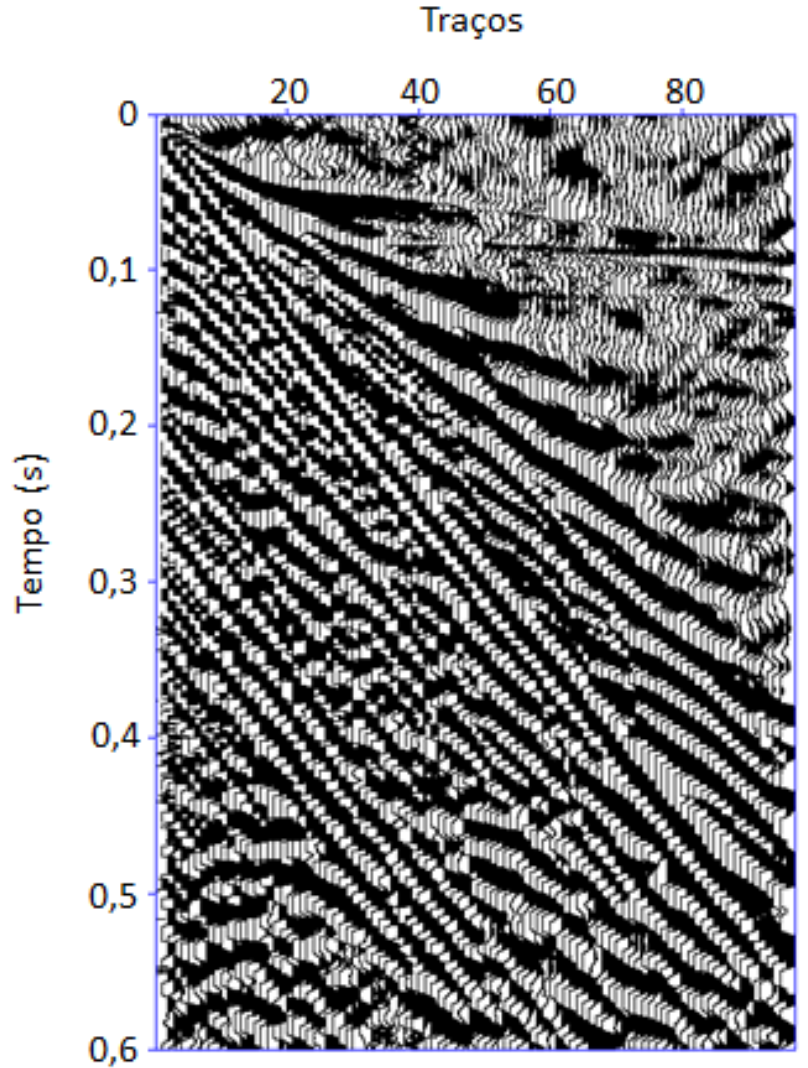

Figura 2: Conjunto de tiro real utilizado para a avaliação do método.

Os valores utilizados para as profundidades das interfaces, velocidades da onda $\mathrm{P}$ assim como os resultados obtidos sobre os dados reais com os seus respectivos intervalos de busca estão expressos na Tabela 4. Os intervalos de busca para a velocidade da onda $S$ foram escolhidos com base no trabalho de Rosa (2008) onde foram realizados ensaios de sísmica multicomponente no mesmo sítio. Os intervalos de busca para as densidades do meio foram escolhidos de acordo com informações litológicas obtidas de furos de sondagens realizados em Porsani et al. (2004) próximos a linha de aquisição.

Tabela 4: Valores de profundidade e velocidade da onda $P$ utilizados no algoritmo de inversão e valores de densidade e velocidade da onda $S$ estimados. Intervalos

\begin{tabular}{|c|c|c|c|c|}
\hline Camada & Profundidade $(\mathrm{m})$ & $V p(m / s)$ & Vs $(\mathrm{m} / \mathrm{s})$ & $\rho\left(\mathrm{Kg} / \mathrm{m}^{3}\right)$ \\
\hline 1 & 3 & 341 & $308(215-500)$ & $1434(1400-2000)$ \\
\hline 2 & 9 & 550 & $475(215-500)$ & $1509(1400-3000)$ \\
\hline 3 & 50 & 2211 & $500(215-500)$ & $1914(1400-3000)$ \\
\hline 4 & - & 4000 & $869(800-2000)$ & $2176(2000-2600)$ \\
\hline
\end{tabular}

Traços

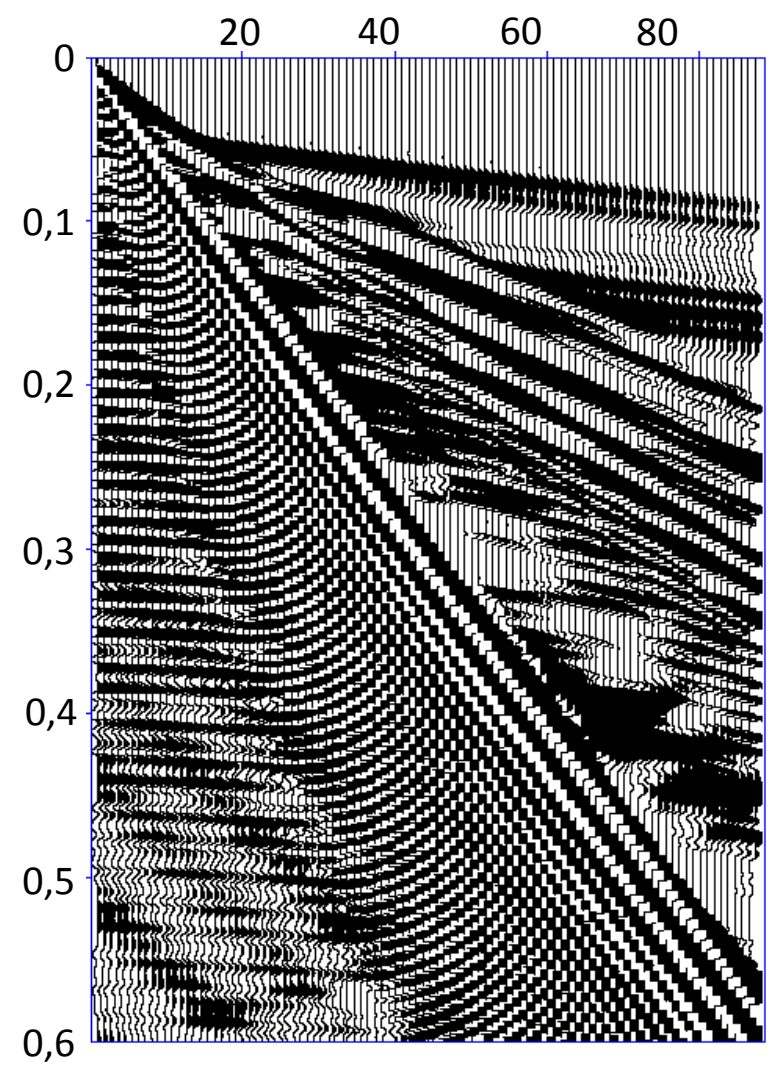

Figura 3: Conjunto de tiro sintético construído com os valores estimados no teste com o dado real.

\section{Discussão e Conclusões}

A aplicação da metodologia se mostrou eficiente desde que os intervalos de busca sejam bem escolhidos. Embora algoritmos genéticos não precisem necessariamente de uma boa estimativa inicial das velocidades e densidades a complexidade do problema torna interessante o conhecimento da geologia da região para uma boa definição dos intervalos de busca.

Nos resultados sintéticos a estimativa das velocidades onda $S$ se saiu melhor. Entretanto, não houve sensibilidade suficiente para uma boa estimativa das densidades. No caso das densidades o intervalo de busca é mais difícil de ser definido. Além disso, a estimativa de ambos os parâmetros abaixo do refletor teve menor precisão.

No caso do dado real é necessário que sejam realizadas experimentações antes da aplicação da metodologia. O fato da wavelet também ser uma incógnita torna necessário que as características da fonte e o espectro do dado sejam considerados. Uma alternativa seria a inclusão da estimativa da wavelet no problema de inversão. 
Comparando o conjunto de tiro real com o sintético gerado a partir dos resultados (Figuras 2 e 3) é possível correlacionar os principais eventos. Apesar disso, existem eventos, de caráter numérico, que não se assemelham com os observados no conjunto de tiro da Figura 2. Além disso, os valores estimados para a onda $S$ ficaram mais baixos que o esperado para a última camada quando comparados aos estimados em dados de refração da onda S em Rosa (2008).

Estes resultados indicam que ainda precisam ser realizados mais testes sobre esta metodologia. Como trabalhos futuros serão testadas diferentes funções objetivos e também a aplicação conjunta com sismogramas de componente radial. A aplicação deste tipo de metodologia merece especial atenção. Nos métodos de refração e de MASW é necessário a utilização de afastamentos em torno de 5 vezes a profundidade dos refletores. Em contrapartida, com as devidas correções na metodologia aplicada é possível realizar estimativas da velocidade da onda $S$ a partir de conjuntos de tiro com janelas curtas em relação a profundidade dos refletores considerados.

\section{Agradecimentos}

Spadini agradece a CNPq pela bolsa de pesquisa.

\section{Referências}

DIOGO, L. A., LE DIAGON, F. M. M. \& PRADO, R. L, 2004. Bedrock imaging using post-critical shallow seismic data, Journal of Applied Geophysics, 57, 1-9.

DITMAR, Pavel et al. Interpretation of shallow refraction seismic data by reflection/refraction tomography. Geophysical Prospecting, Hamburg, Germany, v. 47, p.871-901, 1999.

FABIEN-OUELLET, Gabriel; FORTIER, Richard. Using all seismic arrivals in shallow seismic investigations. Journal Of Applied Geophysics, [s. L.], v. 103, n. [], p.31-42, abr. 2014.

GÉLIS, C; VIRIEUX, J; GRANDJEAN, G. Two-dimensional elastic waveform inversion using

Born and Rytov formulations in the frequency domain. Geophysical Journal International, [S.I.], v. 168, n. [], p.605-633, 2007.

GRANDA, Rafael Garcia. Imageamento do topo rochoso empregando sísmica de reflexão rasa. 2010. $35 \mathrm{f}$. Trabalho de Graduação - Curso de Geofísica, Universidade de São Paulo, São Paulo, 2010.

GRANDJEAN, Gilles. Imaging subsurface objects by seismic P-wave tomography: numerical and experimental validations. Near Surface Geophysics, Orléans, France, v. 4 , n. 5 , p. $275-283$, Oct. 2006.
LIU, Zhiyang; ZHANG, Jie. Elastic full waveform inversion for near surface imaging in CMP domain. Seg Houston 2013 Annual Meeting, Houston, p.1904-1908. 2013.

PORSANI, J. L., BORGES, W. R., ELIS, V. R., DIOGO, L. A., HIODO, F. Y., Marrano, A. \& BIRELLI, C. A., 2004. Investigações geofísicas de superfície e de poço no sítio controlado de geofísica rasa do IAG-USP, Revista Brasileira de Geofísica, vol. 22(3), 245-258.

ROMDHANE A., et al. Inversion of surface waves dispersion in complex structures. In: SYMPOSIUM ON THE APPLICATION OF GEOPHYSICS TO ENVIRONMENTAL AND ENGINEERING PROBLEMS (SAGEEP), 21. 2008, [S.I.]. [S.I.]. [S.I.]: [S.I.], 2008. p. 1 1.

ROMDHANE A., et al. Full waveform inversion of seismic data for 2D shallow structures imagery. 71st Conference And Technical Meeting, Eage, [S. I.], v. 002, n. [], p.1-1, jan. 2009.

ROSA, Daiane Rossi. Ensaios de sísmica rasa multicomponente para ondas S. 2008. 35 f. TCC (Graduação) - Curso de Geofísica, Departamento de Instituto de Astronomia, Geofísica e Ciências Atmosféricas, Universidade de São Paulo, São Paulo, 2008.

THORBECKE, Jan W.; DRAGANOV, Deyan. Finitedifference modeling experiments for seismic interferometry. Geophysics, [s. I.], v. 76, n. 6, p.1-18, nov. 2011.

VESNAVER, Aldo L. et al. Tomographic imaging by reflected and refracted arrivals at the North

Sea. Geophysics, Trieste, Italy, v. 64, n. 6, p.1852-1862, 1999.

VIRIEUX, Jean. P-SV wave propagation in heterogeneous media: Velocity-stress finite-difference method. Geophysics, [S. I.], v. 51, n. 4, p.889-901, abr. 1986. 